\title{
Ventilatory Threshold
}

National Cancer Institute

\section{Source}

National Cancer Institute. Ventilatory Threshold. NCI Thesaurus. Code C123909.

The point during exercise at which pulmonary ventilation becomes disproportionately high with respect to oxygen consumption. 